\title{
Role of postmastectomy radiation therapy in
}

\section{breast cancer patients with $\mathrm{TI}-2$ and I-3 positive lymph nodes}

This article was published in the following Dove Press journal:

OncoTargets and Therapy

9 September 2016

Number of times this article has been viewed

\author{
Yasemin Benderli Cihan' \\ Talha Sarigoz ${ }^{2}$ \\ 'Department of Radiation Oncology, \\ 2Department of General Surgery, \\ Kayseri Training and Research \\ Hospital, Kayseri, Turkey
}

Objective: To evaluate the role of radiotherapy (RT) in overall survival (OS) and disease-free survival in postmastectomy breast cancer patients with tumor size $<5 \mathrm{~cm}$, with $1-3$ involved axillary lymph nodes (T1-2N1).

Patients and methods: We conducted a retrospective study of 89 postmastectomy patients with T1-2N1 disease between 2005 and 2015 at the Radiation Oncology Clinic of Kayseri Training and Research Hospital. Clinicopathologic, demographic, and laboratory findings, as well as treatment regimens were investigated. OS and disease-free survival as well as factors that can be valuable in the prognosis were evaluated.

Results: A total of 89 female patients with an average age of 53 years (range: $30-81$ years) were included in the assessment. Five-year and 10-year local recurrence rates were found to be $6.6 \%$ in the RT group and $7.1 \%$ in the non-RT group. In the RT group, the mean OS was 110.3 months and progression-free survival was 104.4 months. In the non-RT group, the corresponding figures were 104.3 months and 92.1 months, respectively. Statistically significant correlation was observed between RT and the American Joint Committee on Cancer stage $(P<0.001)$, histological type $(P=0.013)$, tumor size $(P<0.001)$, and lymph node metastasis $(P<0.001)$. During the assessment, locoregional recurrence and/or distant metastasis occurred in nine patients $(10 \%)$. Locoregional recurrence was observed mostly in patients with invasive ductal carcinoma, tumor $>3.0 \mathrm{~cm}$ in size, grade II tumors, and perinodal invasion, and who were premenopausal at the time of diagnosis.

Conclusion: In T1-2N1 breast cancer patients who underwent modified radical mastectomy, when the effects of postmastectomy RT were evaluated, there were no differences in terms of OS and progression-free survival. In addition, when subgroup analysis was made, in patients with invasive ductal carcinoma, tumor diameter $>2 \mathrm{~cm}$, three lymph node metastasis, and stage $2 \mathrm{~b}$, postmastectomy RT was seen to be useful.

Keywords: early stage cancer, modified radical mastectomy, radiotherapy, locoregional recurrence, prognostic factors

\section{Introduction}

In the recent years, thanks to advances in diagnosis, cancer typing, staging, and treatment of breast cancer, both disease-free survival (DFS) and overall survival (OS) have improved significantly. With increasing diversity of treatment regimens and pointed importance of locoregional control, radiation therapy (RT) for early stage breast cancer has come to the fore again, so nowadays it is important to clarify which group of patients can benefit from radiation therapy. ${ }^{1-3}$

In the early stages of breast cancer (T1-2N1), surgical resection is the curative treatment modality. The most commonly used surgical method is modified radical Department of Radiation Oncology, Kayseri Training and Research Hospital, Sanayi District, Ataturk Boulevard, Hastane Street, No 78, 38010 Kocasinan, Kayseri, Turkey

Tel +903523368884

Fax +9035232073/3

Email cihany@erciyes.edu.tr 
mastectomy (MRM). However, there is an increasing trend toward breast-conserving surgery lately. ${ }^{2-6}$ According to randomized studies in postmastectomy patients, local recurrence rate varies from $4 \%$ to $40 \%$. Today, chemotherapy is recommended as a standard adjuvant treatment in this group of patients, but which group of patients should be given postmastectomy radiation therapy (PMRT) is still under debate..$^{3-7}$

PMRT aims to eradicate tumor cells on the anterior chest wall and the skin, as well as the regional lymphatics. Tumor size $>5 \mathrm{~cm}$ and four or more positive lymph nodes are the known indications for adjuvant RT after mastectomy. In early stage tumors, RT can be given to improve local control if one of the following criteria is met: young patients at high risk for local recurrence; pathological grade III disease; estrogen receptor (ER) negativity; premenopausal status; lymphovascular invasion; inadequate axillary dissection (ten or fewer lymph node removal); pectoral fascia involvement; or negative surgical margin of $\leq 2 \mathrm{~mm}$. 1,3,5,6,8-10

According to the results of randomized trials, in T1-2N1 breast cancer patients, local recurrence risk (LRR) at 10-year follow-up was reported to be between $8 \%$ and $23 \%$ when no adjuvant RT was given. ${ }^{3-7}$ In the Oxford overview, LRR was found to be $4 \%$ in adjuvant PMRT group and $16 \%$ in nonPMRT group. Particularly in high-risk patients for locoregional recurrence, adjuvant RT decreases that time by at least three times. ${ }^{1-3}$ However, in the recent years, retrospective analysis, patients who underwent MRM, had 1-3 positive lymph nodes, and received adjuvant systemic therapy, LRR was shown to be 5\%-10\%. Today, due to low LRR, administering PMRT to intermediate risk group of patients with T1-2 tumor and 1-3 positive lymph is considered unnecessary. On the other hand, who may benefit from PMRT is a common question. However, from the clinical, pathological, and demographic data, who will most benefit from PMRT is still not clear. Each clinic has its own experience and philosophy of PMRT use. . $^{3,5,6,8-10}$

The purpose of this study is to compare the effect of demographic, clinical, pathological, and biochemical parameters that may have impact on the DFS and OS of patients with T1-2N1 disease whether received PMRT or not, in the Radiation Oncology Clinic.

\section{Patients and methods}

\section{Demographic and clinicopathological characteristics of the patients}

We conducted a retrospective study of 89 postmastectomy patients with T1-2N1 disease between 2005 and 2015 at the Radiation Oncology Clinic of Kayseri Training and Research Hospital. Patients who underwent breast-conserving surgery, had neoadjuvant chemotherapy, or had another concurrent cancer were excluded from the study. This study was designed in accordance with the local ethical principles and the Declaration of Helsinki. The study was approved by the ethics committee of Erciyes University Faculty of Medicine. As the study was retrospective, this committee waived the need for patient consent.

Unfollowed patients and/or patients with missing information were excluded from the assessment. Patients were divided into two groups based on whether they received RT or not. Between these two groups, patient-related characteristics (age, menopausal status, etc), histopathological characteristics (tumor size, tumor location, histology, histological grade, lymphatic, vascular and perineural invasion, estrogen/ progesterone receptor status, human epidermal growth factor receptor 2 [HER2] status, total number of removed lymph nodes, number of metastatic lymph nodes, American Joint Committee on Cancer [AJCC] stage [2010] ${ }^{11}$ ), biochemical parameters (complete blood count, kidney and liver function tests, carcinoembryonic antigen [CEA], cancer antigen 15-3 [CA 15-3]), treatment modalities (surgery, RT, chemotherapy, endocrine therapy), OS, and DFS were compared.

\section{Treatment modalities}

\section{Surgery}

MRM including level I and II axillary dissection was performed in all patients with diagnosis of breast cancer after either fine-needle aspiration biopsy or excisional biopsy. Pathological staging was done according to the AJCC breast cancer staging manual 2010.

\section{Chemotherapy}

Adjuvant therapy (chemotherapy or hormonotherapy) was given after surgical resection, considering features such as performance status, age, and comorbidities. Adjuvant chemotherapy was given for 3-5 weeks to patients with tumor size $1 \mathrm{~cm}$ or larger or one or more axillary lymph node involvement, Eastern Cooperative Oncology Group performance status 0-2, and normal liver, heart, kidney, and bone marrow functions. Patients underwent one of the following regimes: cyclophosphamide, methotrexate, and 5-fluorouracil; cyclophosphamide, doxorubicin, and 5-fluorouracil; cyclophosphamide, epirubicin, and 5-fluorouracil; doxorubicin and cyclophosphamide; or docetaxel.

\section{Radiotherapy}

Before RT, risk and benefits of PMRT were presented to patients, and their preference was considered. Particularly, if a patient had two or more of these factors - namely age $<40$ years, tumor size $>2 \mathrm{~cm}$, ER status (-), triple (-) 
hormone receptor status, HER2 (3+), LVI status (+), three lymph node metastasis, nuclear grade 3 , extracapsular lymph node invasion - RT was performed after chemotherapy. Conformal and conventional plans were created for all patients. A classic simulator was used for conventional planning and a computed tomography (CT) simulator was used for conformal planning. Cobalt-60 or 6-MV photon beams were selected for the treatment. Postmastectomy adjuvant RT was given from two tangential fields at 1.8-2 Gy/d fraction, 5 days a week, and at a total dose of 46-50 Gy; cases with near surgical margins or positive margins and extracapsular invasion were given additional doses at $10 \mathrm{~Gy} / 1.8-2 \mathrm{~Gy}$ fraction. Radiation therapy field composed of the chest wall, the internal mammary node, and the supraclavicular area. Level III axillary nodal region was included in some of the regimens but levels I and II were excluded from the RT field. Patients who are younger than 40 years, those with tumor size $>2 \mathrm{~cm}$, negative ER status, positive LVI status, extracapsular lymph node invasion, and higher nuclear grades ( 2 or 3 ) were evaluated in the high-risk category and PMRT was used.

\section{Hormone therapy}

Following RT and/or chemotherapy treatment, hormone therapy was given to cases that were positive for estrogen and/or progesterone receptors. Tamoxifen \pm LHRH (luteinizing hormone releasing hormone) analogs in premenopausal patients or tamoxifen or aromatase inhibitors in postmenopausal patients were given for 5 years.

\section{Biochemical parameters}

Preoperative white blood cell, hemoglobin, and platelet counts, neutrophil/lymphocyte ratio, platelet/lymphocyte ratio, blood glucose, blood urea nitrogen, creatinine, aspartate aminotransferase, gamma-glutamyl transferase, lactate dehydrogenase ( $\mathrm{LDH}$ ), calcium, total protein, albumin, carcinoembryonic antigen (CEA), and CA 15-3 were evaluated. The patients were divided into two groups according to the median value of neutrophil/lymphocyte ratio and platelet/ lymphocyte ratio (low: 3 equal and $<3$ or higher: $>3$ and low: 160 equal and $<160$ or high: $\geq 161$, respectively). While evaluating other blood parameters (except for white blood cell and platelet counts), they were classified as higher or normal according to reference range.

\section{Assessment after treatment and follow-up}

During RT, patients were called to the policlinic control once a week. In case of necessity, they were followed closely and more frequently for side effects. Response to treatment was assessed according to World Health Organization criteria.
Patients without significant side effects after the end of treatment were called to first control at first month. Then patients were controlled every 3 months until 2 years, every 6 months until 5 years, and then annually after 5 years. Every 6 months, physical examination, complete blood count, biochemistry panel, CA 15-3 and CEA, annual mammography, abdominal ultrasound, and chest X-ray, and, if necessary, tests such as CT scan, magnetic resonance imaging, and whole-body bone scans were performed.

\section{Statistical analysis}

The Statistical Package for the Social Sciences (SPSS) 15.0 software (IBM Corporation, Armonk, NY) was used for the statistical analysis. While analyzing data, as well as descriptive statistical methods (mean, standard deviation), chi-square test, Fisher's exact test, and Student's $t$-test were used for the comparison of qualitative data. While OS was calculated using the time from diagnosis to death, DFS was calculated as the time from diagnosis until diagnosis with recurrence (ipsilateral breast or chest wall recurrence, isolated axillary, supraclavicular or internal mammary node recurrence), or metastasis. Survival was calculated using Kaplan-Meier analysis, univariate analysis was performed using Log-rank, and multivariate analysis was performed using the Cox regression analysis. $P$-values $<0.05$ were considered statistically significant.

\section{Results}

Five patients due to lack of file information and 1 patient due to the development of second primary cancer during the follow-up were excluded from the study. A total of 89 female patients with an average age of 53 years (range: 30-81 years) were included in the assessment. Of all the patients, $71.9 \%$ were between 41 years and 65 years of age, $55.1 \%$ were postmenopausal, $83 \%$ had invasive ductal carcinoma, $82 \%$ had stage IIb disease, 33.7\% had tumor size of 2-3 cm, 42.7\% had one metastatic lymph node, $43.82 \%$ had grade II tumor, $70.8 \%$ had perinodal involvement, 75.3\% had lymphovascular invasion, 68.5\% were ER positive, $61.8 \%$ were progesterone receptor positive, and $31.5 \%$ were positive for HER2. Ninety-two percent of the patients received chemotherapy, $70.8 \%$ hormone therapy, and $68.5 \%$ RT. The chemotherapy regimens were as follows: 5-fluorouracil, epirubicin, cyclophosphamide; and doxorubicin, cyclophosphamide.

In Table 1, we compare the clinical, pathological, and blood parameters of the patients that received RT or not by the Pearson chi-square test. Statistically significant correlation was observed between RT with the AJCC stage $(P<0.001)$, 
Table I Comparison of clinical, pathological, and biochemical parameters of postmastectomy patients with $\mathrm{TI}-2$ disease and I-3 positive lymph nodes $(n=89)$

\begin{tabular}{|c|c|c|c|c|c|c|c|}
\hline \multirow{2}{*}{\multicolumn{4}{|c|}{$\begin{array}{l}\text { parameters of postmastectomy patients with TI-2 disease and } \\
\mathrm{I}-3 \text { positive lymph nodes }(n=89)\end{array}$}} & & \multirow{3}{*}{$\begin{array}{l}\text { RT not received } \\
(\mathbf{N}=\mathbf{2 8})\end{array}$} & \multirow{3}{*}{$\begin{array}{l}\text { RT received } \\
(\mathbf{N}=6 \mathrm{I})\end{array}$} & \multirow{3}{*}{$\begin{array}{l}\boldsymbol{P} \text {-value } \\
(<\mathbf{0 . 0 5}) \\
0.337\end{array}$} \\
\hline & & & & & & & \\
\hline & $\begin{array}{l}\mathrm{RT} \text { not received } \\
(\mathrm{N}=\mathbf{2 8})\end{array}$ & $\begin{array}{l}\text { RT received } \\
(\mathbf{N}=6 I)\end{array}$ & $\begin{array}{l}P \text {-value } \\
(<0.05)\end{array}$ & $\begin{array}{l}\text { Lymphovascular } \\
\text { invasion }\end{array}$ & & & \\
\hline Age (years) & & & 0.376 & No & 9 & 13 & \\
\hline$\leq 44$ & 4 & 13 & & Yes & 19 & 48 & \\
\hline$>44$ & 24 & 48 & & Chemotherapy & & & 0.813 \\
\hline Menonausal status & 24 & 48 & & Yes & 26 & 56 & \\
\hline Menopausal status & & & 0.516 & No & 2 & 5 & \\
\hline Premenopausal & 14 & 26 & & Hormone therapy & & & 0.963 \\
\hline Postmenopausal & 14 & 35 & & Yes & 20 & 43 & \\
\hline Tumor localization & & & 0.988 & No & 8 & 16 & \\
\hline Right & 15 & 34 & & 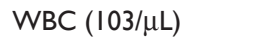 & & & 0.756 \\
\hline Left & 13 & 27 & & $\leq 8.0$ & 14 & 33 & \\
\hline AJCC stage & & & $<0.001$ & $>8.01$ & 14 & 28 & \\
\hline Stage IIA & 13 & $\begin{array}{l}3 \\
58\end{array}$ & & Hemoglobin (g/dL) & & & 0.96 \\
\hline $\begin{array}{l}\text { Stage IIB } \\
\text { Histological type }\end{array}$ & 15 & 38 & & $\leq 12.0$ & 1 & 8 & \\
\hline $\begin{array}{l}\text { Histological type } \\
\text { Invasive ductal }\end{array}$ & 18 & 56 & 0.013 & $>12.01$ & 27 & 53 & \\
\hline Inflammatory & 5 & 4 & & Platelet $(103 / \mu \mathrm{L})$ & & & 0.401 \\
\hline Other & 5 & I & & $\leq 300,000$ & 22 & 50 & \\
\hline Tumor size $(\mathrm{T})$ & & & $<0.001$ & $>301,000$ & 6 & 11 & \\
\hline I & 14 & 3 & & NLR & & & 0.527 \\
\hline$\|$ & 14 & 58 & & $\leq 3.0$ & 19 & 32 & \\
\hline Tumor diameter & & & 0.011 & $>3.1$ & 9 & 29 & \\
\hline$\leq \mathrm{I} \mathrm{cm}$ & 5 & I & & PLR & & & 0.545 \\
\hline $1.0 \mathrm{I}-2 \mathrm{~cm}$ & 6 & 2 & & $\leq 160,000$ & 21 & 39 & \\
\hline $2.0 \mathrm{I}-3 \mathrm{~cm}$ & 9 & 21 & & $>161,000$ & 7 & 22 & \\
\hline $3.0 \mathrm{I}-4 \mathrm{~cm}$ & 5 & 21 & & Glucose (mg/dL) & & & 0.736 \\
\hline $4.0 \mathrm{I}-5 \mathrm{~cm}$ & 3 & 16 & & $\leq 105$ & 18 & 35 & \\
\hline Lymph node metastasis & & & $<0.001$ & $>106$ & 10 & 26 & \\
\hline I lymph node & 23 & 15 & & Blood urea nitrogen & & & 0.546 \\
\hline 2 lymph nodes & 5 & 32 & & $(\mathrm{mg} / \mathrm{dL})$ & & & \\
\hline 3 lymph nodes & 0 & 14 & & $\leq 26$ & 25 & 57 & \\
\hline Total number of & & & 0.573 & $>27$ & 3 & 40 & \\
\hline dissected lymph nodes & & & & Creatinine $(\mathrm{mg} / \mathrm{dL})$ & & & 0.2 \\
\hline$\leq 10$ & 5 & 13 & & $\leq 1.3$ & 27 & 59 & \\
\hline $11-16$ & 9 & 16 & & $>1.31$ & 1 & 2 & \\
\hline$\geq 17$ & 14 & 32 & & AST (U/L) & & & 0.953 \\
\hline Histological grade & & & 0.218 & $\leq 34$ & 25 & 54 & \\
\hline I & 9 & 10 & & $>35$ & 3 & 7 & \\
\hline II & 13 & 26 & & GGT (U/L) & & & 0.727 \\
\hline III & 5 & 18 & & $\leq 36$ & 24 & 51 & \\
\hline Unknown & 1 & 7 & & $>37$ & 4 & 10 & \\
\hline ER status & & & 0.281 & $\operatorname{ALP}(\mathrm{U} / \mathrm{L})$ & & & 0.989 \\
\hline Positive & 17 & 44 & & $\leq 300$ & 27 & 58 & \\
\hline Negative & 11 & 17 & 0333 & $>301$ & 1 & 2 & \\
\hline $\begin{array}{l}\text { PR status } \\
\text { Negative }\end{array}$ & 8 & 26 & & LDH (U/L) & & & $0.04 I$ \\
\hline Positive & 20 & 35 & & $\leq 280$ & 20 & 50 & \\
\hline HER2 & & & 0.293 & $>281$ & 8 & 9 & \\
\hline Negative & 6 & 22 & & Calcium (mg/dL) & & & 0.733 \\
\hline Positive & 22 & 37 & & $\leq 10$ & 23 & 51 & \\
\hline Unknown & 0 & 2 & & $>10.1$ & 4 & 10 & \\
\hline Perinodal involvement & & & 0.447 & Total protein $(\mathrm{g} / \mathrm{dL})$ & & & 0.154 \\
\hline No & 9 & 17 & & $\leq 8.3$ & 21 & 54 & \\
\hline Yes & 19 & 44 & & $>8.31$ & 4 & 3 & \\
\hline
\end{tabular}

Table I (Continued) 
Table I (Continued)

\begin{tabular}{|c|c|c|c|}
\hline & $\begin{array}{l}\text { RT not received } \\
(\mathrm{N}=\mathbf{2 8})\end{array}$ & $\begin{array}{l}\text { RT received } \\
(\mathrm{N}=6 \mathrm{I})\end{array}$ & $\begin{array}{l}P \text {-value } \\
(<0.05)\end{array}$ \\
\hline Albumin (g/dL) & & & 0.271 \\
\hline$\leq 3.5$ & 26 & 55 & \\
\hline$>3.6$ & I & 6 & \\
\hline CEA (ng/mL) & & & 0.511 \\
\hline$\leq 3$ & 24 & 46 & \\
\hline$>3.1$ & 4 & 15 & \\
\hline CA I5-3 (U/mL) & & & 0.155 \\
\hline$\leq 31.6$ & 28 & 57 & \\
\hline$>31.7$ & 0 & 4 & \\
\hline
\end{tabular}

Abbreviations: AJCC, American Joint Committee on Cancer; ER, estrogen receptor; PR, progesterone receptor; HER2, human epidermal growth factor receptor 2; WBC, white blood cell; NLR, neutrophil/lymphocyte ratio; PLR, platelet/lymphocyte ratio; AST, aspartate aminotransferase; GGT, gamma-glutamyl transferase; ALP, alkaline phosphatase; LDH, lactate dehydrogenase; CEA, carcinoembryonic antigen; CA 15-3, cancer antigen 15-3; RT, radiotherapy.

histological type $(P=0.013)$, tumor size $(P<0.001)$, lymph node metastasis $(P<0.001)$, and LDH $(P=0.041)$.

Five-year and 10-year local recurrence rate in the RT group was $6.6 \%$ and in the non-RT group $7.1 \%$. In Table 2 , for all the patients, the clinicopathological features with locoregional recurrence and/or distant metastasis are given. Locoregional recurrence was seen mostly in those with invasive ductal carcinoma, tumors $>3.0 \mathrm{~cm}$ in diameter, grade II tumor, and perinodal invasion, and those who were premenopausal.

Table 2 Clinical and pathological features of patients with locoregional recurrence and/or distant metastasis

\begin{tabular}{lllllllllll}
\hline $\begin{array}{l}\text { Patient } \\
\text { characteristics }\end{array}$ & \multicolumn{1}{ll}{ RT received } & & & & & & & \multicolumn{3}{l}{$\begin{array}{l}\text { RT not } \\
\text { received }\end{array}$} \\
\hline Age (years) & 55 & 68 & 47 & 52 & 50 & 44 & 36 & 46 & 50 \\
Menopausal status & Post & Post & Pre & Pre & Post & Pre & Pre & Pre & Post \\
Histological type & IDC & IDC & IDC & IDC & IDC & IDC & IDC & IDC & IDC \\
Tumor size (T) & 3.5 & 5 & 3.5 & 3.5 & 2.3 & 3.6 & 4.5 & 2 & 2.3 \\
Lymph node & I & I & I & I & I & I & 2 & I & I \\
metastasis & & & & & & & & & \\
Histologic grade & I & III & II & II & I & II & II & II & I \\
ER status & - & + & + & + & + & - & + & - & + \\
PR status & - & + & - & + & + & - & + & + & + \\
HER2 & - & - & - & - & - & - & - & - & - \\
Perinodal & + & + & + & + & + & - & + & - & + \\
involvement & & & & & & & & & \\
Lymphovascular & + & + & - & - & - & + & + & - & - \\
invasion & & & & & & & & & \\
Chemotherapy & + & - & + & + & + & + & + & + & + \\
Hormonotherapy & - & + & + & + & + & - & + & + & + \\
Distant metastasis & - & + & - & - & - & + & + & - & - \\
\hline
\end{tabular}

Abbreviations: RT, radiotherapy; Post, postmenopausal; Pre, premenopausal; IDC, invasive ductal carcinoma; ER, estrogen receptor; PR, progesterone receptor; HER2, human epidermal growth factor receptor 2.
In Table 3, the factors influencing OS, PFS, mean OS, and mean PFS values are given. In the RT group, the mean OS was 110.3 months, PFS was 104.4 months; in non-RT group they were 104.3 and 92.1 months, respectively $(P=0.648$, $P=0.482$ ). During the assessment, locoregional recurrence and/or distant metastasis were found in nine patients (10\%). Six patients showed locoregional recurrence, one patient bone metastasis, one patient liver metastasis, and one patient both liver metastasis and locoregional recurrence. Six (6.7\%) patients died. Although both OS and PFS were longer in patients with stage $2 \mathrm{~A}$ tumors, positive hormone receptors, normal levels of CEA and LDH, and endocrine treatment, the level of statistical significance was not reached (Table 3). According to risk factors, in univariate and multivariate analyses, there were no statistically significant factors affecting OS and PFS.

\section{Discussion}

Patients with breast cancer, which was known as a fatal disease and was treated by cauterization by Egyptian physicians in the early ages, underwent intensive surgery until the end of the 19th century. In the early 20th century, it became clear that the prognosis differed from patient to patient, and therefore different treatment methods have been discussed. In parallel, due to the developments in hardware and information technology and implementation of widespread screening programs during the 21 st century, benefits of RT have started to be questioned in patients with $\mathrm{T} 1-2 \mathrm{~N} 1$ disease after the standard use of adjuvant chemotherapy and hormone therapy. RT holds an important place in the multimodal treatment of breast cancer. In the meta-analysis, both for local control and for survival, RT has been found beneficial. According to the consensus opinions and guidelines, patients with more than four lymph node metastasis or stage $\mathrm{T} 3 \mathrm{~N}+$ or positive surgical margins are included in the "white zone", and PMRT is indicated. Patients with tumor $<5 \mathrm{~cm}$ without axillary metastases and negative surgical margins are included in the "black zone", and PMRT may not be applied. Patients with T1-2 tumor and 1-3 positive lymph nodes fall in the "gray zone", and indication of PMRT in this group is not clarified.

In this study, LRR in PMRT group was $6.6 \%$ and in nonPMRT group $7.1 \%$. The longer the follow-up periods, the higher were the postmastectomy local recurrence rates: at 10 years it was $16 \%$ and at 15 years it was $33 \% .{ }^{12}$ Data from randomized trials of MD Anderson, the Eastern Cooperative Oncology Group, and the National Surgical Adjuvant Breast and Bowel Project indicate that in patients with T1-2N1 disease that did 
Table 3 Overall survival and disease-free survival according to clinicopathological and laboratory parameters

\begin{tabular}{|c|c|c|c|c|}
\hline $\begin{array}{l}\text { Patient and tumor } \\
\text { characteristics }\end{array}$ & $\begin{array}{l}\text { Overall survival, survival } \\
\text { month mean }(95 \% \mathrm{Cl})\end{array}$ & $P$-value $(<0.05)$ & $\begin{array}{l}\text { Disease-free survival, survival } \\
\text { month mean }(95 \% \mathrm{Cl})\end{array}$ & $\boldsymbol{P}$-value $(<0.05)$ \\
\hline Age (years) & & 0.149 & & 0.452 \\
\hline$\leq 44$ & $45.6(37.4-53.9)$ & & $43.3(36.4-50.3)$ & \\
\hline$>44$ & I I $2.2(96.4-128.1)$ & & $105.2(87.3-123.0)$ & \\
\hline Menopausal status & & 0.256 & & 0.291 \\
\hline Premenopausal & $100.8(73.4-128.3)$ & & $100.2(76.9-123.49)$ & \\
\hline Postmenopausal & $95.6(79.2-122.1)$ & & $94.5(77.6-111.5)$ & \\
\hline Tumor localization & & 0.444 & & 0.95 \\
\hline Left & $104.3(82.0-126.7)$ & & $111.8(97.0-126.6)$ & \\
\hline Right & $84.9(72.7-97.1)$ & & $74.7(57.2-92.3)$ & \\
\hline AJCC stage & & 0.418 & & 0.638 \\
\hline Stage IIA & 109.1 (84.8-133.4) & & $100.7(57.1-\mid 44.3)$ & \\
\hline Stage IIB & $92.0(76.5-107.1)$ & & $89.7(74.6-104.9)$ & \\
\hline Histology type & & 0.252 & & 0.773 \\
\hline Invasive ductal & - & & - & \\
\hline \multicolumn{5}{|l|}{ Inflammatory } \\
\hline \multicolumn{5}{|l|}{ Other } \\
\hline Tumor size $(T)$ & & 0.565 & & 0.548 \\
\hline I & - & & 107.5 (72.9-|42.29) & \\
\hline II & & & $89.5(74.3-104.7)$ & \\
\hline Tumor diameter & & 0.565 & & 0.43 \\
\hline$\leq \mathrm{I} \mathrm{cm}$ & - & & - & \\
\hline \multicolumn{5}{|l|}{$1.01-2 \mathrm{~cm}$} \\
\hline \multicolumn{5}{|l|}{$2.0 \mathrm{I}-3 \mathrm{~cm}$} \\
\hline \multicolumn{5}{|l|}{$3.0 \mathrm{I}-4 \mathrm{~cm}$} \\
\hline \multicolumn{5}{|l|}{$4.01-5 \mathrm{~cm}$} \\
\hline Lymph node status & & 0.593 & & 0.096 \\
\hline I lymph node metastasis & $104.8(83.3-126.2)$ & & $105.7(79.6-131.8)$ & \\
\hline $\mathrm{I}-2$ lymph node metastasis & $85.5(69.4-101.6)$ & & $84.2(72.6-95.8)$ & \\
\hline 3 lymph node metastasis & $63.1(44.2-82.0)$ & & $50.5(28.1-72.8)$ & \\
\hline Total number of dissected lymph nodes & & 0.300 & & 0.497 \\
\hline$\leq 10$ & - & & II3.I (87.2-139.1) & \\
\hline $11-16$ & & & $108.7(83.9-133.5)$ & \\
\hline$>17$ & & & $80.7(69.1-92.2)$ & \\
\hline Histological grade & & 0.349 & & 0.815 \\
\hline I & - & & - & \\
\hline \multicolumn{5}{|l|}{ II } \\
\hline \multicolumn{5}{|l|}{ III } \\
\hline \multicolumn{5}{|l|}{ Unknown } \\
\hline ER status & & 0.4 & & 0.248 \\
\hline Negative & $97.8(68.6-127.0)$ & & $95.5(66.6-124.4)$ & \\
\hline Positive & I I $2.8(95.1-130.5)$ & & $107.4(85.1-129.7)$ & \\
\hline PR status & & 0.587 & & 0.964 \\
\hline Negative & $106.5(82.7-130.4)$ & & $109.2(90.0-128.4)$ & \\
\hline Positive & $114.5(101.7-127.2)$ & & $95.3(65.9-124.8)$ & \\
\hline HER2 & & 0.209 & & 0.657 \\
\hline Negative & - & & $64.1(53.6-74.6)$ & \\
\hline Positive & & & III.5 (95.4-127.7) & \\
\hline Unknown & & & $72.7(46.6-98.7)$ & \\
\hline Perinodal involvement & & 0.963 & & 0.051 \\
\hline No & $85.7(73.8-97.6)$ & & - & \\
\hline Yes & $103.8(81.7-125.9)$ & & & \\
\hline Lymphovascular invasion & & 0.379 & & 0.268 \\
\hline No & $106.5(84.6-128.5)$ & & $90.1(59.3-121.0)$ & \\
\hline Yes & $91.5(73.8-109.2)$ & & $99.6(90.4-108.7)$ & \\
\hline Chemotherapy & & 0.695 & & 0.354 \\
\hline Yes & III.8 (96.8-126.8) & & & \\
\hline No & $83.8(68.8-98.8)$ & & & \\
\hline
\end{tabular}


Table 3 (Continued)

\begin{tabular}{|c|c|c|c|c|}
\hline $\begin{array}{l}\text { Patient and tumor } \\
\text { characteristics }\end{array}$ & $\begin{array}{l}\text { Overall survival, survival } \\
\text { month mean }(95 \% \mathrm{Cl})\end{array}$ & $\boldsymbol{P}$-value $(<0.05)$ & $\begin{array}{l}\text { Disease-free survival, survival } \\
\text { month mean }(95 \% \mathrm{Cl})\end{array}$ & $P$-value $(<0.05)$ \\
\hline Radiotherapy & & 0.648 & & 0.482 \\
\hline Yes & I I 0.3 (91.3-129.2) & & $104.4(73.5-122.8)$ & \\
\hline No & $92.1(56.4-127.9)$ & & $104.3(86.1-135.2)$ & \\
\hline Hormone therapy & & 0.97 & & 0.102 \\
\hline Yes & II $5.2(103.1-127.4)$ & & $119.6(110.2-128.9)$ & \\
\hline No & $96.9(65.7-128.2)$ & & $94.7(67.1-122.4)$ & \\
\hline WBC $(103 / \mu \mathrm{L})$ & & 0.495 & & 0.145 \\
\hline$\leq 8.0$ & II I.8 (88. I-I35.5) & & $92.3(65.8-118.9)$ & \\
\hline$>8.1$ & $89.2(71.9-106.6)$ & & $101.7(92.2-111.2)$ & \\
\hline Hemoglobin (g/dL) & & 0.443 & & 0.203 \\
\hline$\leq 12.0$ & - & & 46.1 (27.5-64.7) & \\
\hline$>12.1$ & & & I I $2.5(99.9-125.2)$ & \\
\hline 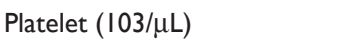 & & 0.763 & & 0.855 \\
\hline$\leq 300,000$ & $108.0(90.1-126.0)$ & & $109.0(93.5-124.4)$ & \\
\hline$>301,000$ & $100.9(59.2-142.6)$ & & $83.9(35.8-131.9)$ & \\
\hline NLR & & 0.555 & & 0.101 \\
\hline$\leq 3.0$ & $88.6(70.6-106.5)$ & & $100.3(88.7-|1| 2.0)$ & \\
\hline$>3.1$ & $116.5(101.2-131.7)$ & & $94.8(68.9-120.7)$ & \\
\hline PLR & & 0.345 & & 0.395 \\
\hline$\leq 160.000$ & $90.8(75.8-105.8)$ & & $96.2(84.1-108.4)$ & \\
\hline$>161.000$ & $108.2(74.5-14 \mid .8)$ & & $90.5(54.0-127.0)$ & \\
\hline Glucose (mg/dL) & & 0.104 & & 0.287 \\
\hline$\leq 105$ & - & & I I $0.3(92.2-128.5)$ & \\
\hline$>106$ & & & $96.3(67.2-125.4)$ & \\
\hline Blood urea nitrogen (mg/dL) & & 0.691 & & 0.31 \\
\hline$\leq 26$ & $105.7(86.5-125.0)$ & & - & \\
\hline$>27$ & $89.9(56.8-123.0)$ & & & \\
\hline Creatinine (mg/dL) & & 0.601 & & 0.51 \\
\hline$\leq 1.3$ & - & & - & \\
\hline$>1.31$ & & & & \\
\hline AST (U/L) & & 0.237 & & 0.212 \\
\hline$\leq 34$ & - & & - & \\
\hline$>35$ & & & & \\
\hline GGT (U/L) & & 0.857 & & 0.249 \\
\hline$\leq 36$ & - & & - & \\
\hline$>37$ & & & & \\
\hline ALP (U/L) & & $0.84 I$ & & 0.74 \\
\hline$\leq 300$ & - & & - & \\
\hline$>301$ & & & & \\
\hline LDH (U/L) & & 0.165 & & 0.533 \\
\hline$\leq 280$ & I I 2.6 (94.9-130.2) & & $108.3(92.1-124.5)$ & \\
\hline$>281$ & $64.9(53.9-75.9)$ & & $69.4(61.0-77.8)$ & \\
\hline Calcium (mg/dL) & & 0.672 & & 0.27 \\
\hline$\leq 10$ & $93.0(77.2-108.8)$ & & $94.6(82.0-107.1)$ & \\
\hline$>10.1$ & $103.8(73.7-133.8)$ & & $92.9(59.0-126.9)$ & \\
\hline Total protein (g/dL) & & 0.279 & & 0.265 \\
\hline$\leq 8.3$ & II 0.6 (93.0-128.2) & & - & \\
\hline$>8.31$ & $86.2(45.6-126.8)$ & & & \\
\hline Albumin $(g / d L)$ & & 0.127 & & 0.935 \\
\hline$\leq 3.5$ & $69.8(44.7-94.9)$ & & $81.2(62.7-99.8)$ & \\
\hline$>3.6$ & $109.3(90.7-128.0)$ & & $104.2(85.6-122.7)$ & \\
\hline CEA $(\mathrm{ng} / \mathrm{mL})$ & & 0.89 & & 0.704 \\
\hline$\leq 3$ & $109.8(90.6-128.9)$ & & $107.1(89.0-125.1)$ & \\
\hline$>3.1$ & $105.7(80.1-131.3)$ & & $104.6(77.1-132.2)$ & \\
\hline CA I5-3 (U/mL) & & 0.324 & & 0.346 \\
\hline$\leq 31.6$ & - & & - & \\
\hline$>31.7$ & & & & \\
\hline
\end{tabular}

Abbreviations: AJCC, American Joint Committee on Cancer; ER, estrogen receptor; PR, progesterone receptor; HER2, human epidermal growth factor receptor 2; WBC, white blood cell; NLR, neutrophil/lymphocyte ratio; PLR, platelet/lymphocyte ratio; AST, aspartate aminotransferase; GGT, gamma-glutamyl transferase; ALP, alkaline phosphatase; LDH, lactate dehydrogenase; CEA, carcinoembryonic antigen; CA 15-3, cancer antigen 15-3; Cl, confidence interval. 
not receive PMRT, LRR was $\sim 30 \%$ in a 10 -year follow-up. According to the reports of Danish trials, the British Columbia trials, and the Early Breast Cancer Trialists' Collaborative Group (EBCTCG) meta-analysis, it was 20\%. ${ }^{3-7}$

In this work-up, there was significant difference between the AJCC stage, histologic type, tumor size, and lymph node metastasis when the two groups were compared. According to the 2011 St Gallen Consensus Conference ${ }^{13}$ on early breast cancer, tumor size is an independent prognostic factor particularly if lymph node metastasis is negative. ${ }^{13}$ For larger tumors, disease recurrence is found to increase and clinical course is seen to deteriorate. According to the Memorial Sloan Kettering Cancer Center data, tumors $<1 \mathrm{~cm}$ have a 20 -year recurrence rate of $12 \% .{ }^{14}$ Gebauer et $\mathrm{al}^{15}$ studied 1,162 breast cancer patients and reported that in patients with tumors $>2 \mathrm{~cm}$, DFS and OS were worse than those with smaller tumors. In the American National Cancer Institute Surveillance Epidemiology and End Results (SEER) program, 24,740 patients were examined, and it was found that the more the lymph node involvement, the higher the recurrence risk and the lower the survival, and this relation was found to be proportional to the tumor size. ${ }^{16}$ Shen et al $^{17}$ studied $1,030 \mathrm{~T} 1-2 \mathrm{~N} 1$ breast cancer patients and reported worse prognosis when lymph node involvement was high. Tumor size and axillary lymph node status still remain among the most important prognostic factors. In this study, similar to previous reports, we found that with increasing number of lymph nodes and larger tumor size, the recurrence risk was higher.

In our study, the 10-year locoregional recurrence rate was $6.6 \%$ in the group receiving RT and $7.1 \%$ in the other group. The group receiving $\mathrm{RT}$ showed higher OS and PFS. When the two groups were compared, there was a significant relationship between the AJCC stage, histologic type, tumor size, and number of metastatic lymph nodes. In addition, locoregional recurrence was observed mostly in patients with invasive ductal carcinoma, tumor $>3.5 \mathrm{~cm}$ in size, grade II tumors, negative hormone receptors, and perinodal invasion, and those who were premenopausal at the time of diagnosis. For patients with some of these features, locoregional recurrence risk was high even though they received RT. Today, it is known that estimation of biological behavior and anatomical presence of tumors is not appropriate in RT planning. Therefore, in the decision-making process of RT, in order to improve the accuracy of survival and metastatic disease prediction, risk factors are being increasingly used. ${ }^{4,5}$ Truong et al studied 821 postmastectomy patients with $\mathrm{T} 1-2 \mathrm{~N} 1$ disease for whom adjuvant RT could not be given and found that the 10-year isolated LRR and LRR with or without simultaneous distant recurrence rate was $12.7 \%$ and $15.9 \%$, respectively. In the subgroup analysis of patients with 1-3 positive lymph nodes, they reported a 10 -year LRR of $25 \%$ or more if there was at least one risk factor such as below 45 years of age, T2 tumor, grade 3 tumor, positive the lymph nodes $>25 \%$ of the total lymph nodes, medial tumor location, or negative ER. ${ }^{2}$ In their study, Yang et al reported that RT reduced LRR and improved survival in patients with negative ER and lymphovascular invasion. ${ }^{4}$ In a randomized study including 983 subjects with T1-2 tumors and 1-3 positive nodes, the 10-year risk of LRR with or without simultaneous distant recurrence was $12 \%$. In the multivariate analysis, a larger tumor size, increasing number of positive nodes, and negative ER status were independent predictors of LRR. ${ }^{18}$ In the work of Wallgren et al, ${ }^{19}$ the factors associated with increased LRR in premenopausal patients were high histologic grade and vascular invasion; in postmenopausal patients they were tumor size $>2 \mathrm{~cm}$ and high histologic grade. According to other studies including patients with T1-2N1 disease, close or positive surgical margins, gross extracapsular extension, dissection of fewer than ten nodes, young age, LVI, nuclear grade, ER status, tumor size, and medial tumor location were associated with higher LRR. RT was suggested for this group of patients. ${ }^{1,6,8,9}$ Adjuvant RT reduces LRR at least by fourfold in patients who are at high risk for recurrence. For example, in a previous analysis of the MD Anderson patients with T1-2 disease and 1-3 positive lymph nodes, it was demonstrated that PMRT reduced the 10-year LRR risk from $13 \%$ to $3 \%$ $(P<0.03) .^{2,4,20}$ Overgaard et $\mathrm{al}^{21}$ followed 114 patients that received only $\mathrm{CT}$ or $\mathrm{CT} / \mathrm{RT}$ and reported an LRR of $23 \%$ and $5 \%$, respectively. The ongoing MRC/EORTC SUPREMO trial is expected to find out in which group of patients, patients who underwent MRM with pT1N0M0 or pT2N0-1M0, PMRT will be beneficial. ${ }^{20}$ In this study, our results were similar to the results of those that evaluated the risk factors in relation to LRR. Lack of significant results for some of the risk factors that we evaluated can be explained by patient selection, the relatively small group, and systemic therapy.

Although there was no statistically significant difference, OS and DFS were longer in patients with normal levels of preoperative LDH and CEA. When the groups that received RT and did not receive it were compared for blood parameters, they showed no difference except LDH level. In patients with breast cancer, both clinical and laboratory findings have been analyzed in relation to OS and DFS. Multiple studies have shown that chronic inflammation launched the process of carcinogenesis and acted as a predisposing factor for cancer. ${ }^{22,23}$ Total serum LDH levels provide information about both inflammation and tissue damage. Serum LDH level has been shown to be a prognostic marker in many malignancies such 
as lymphoma and metastatic melanoma. ${ }^{23}$ However, there are few studies showing whether pretreatment blood parameters carry any prognostic value.

\section{Conclusion}

When RT group and non-RT group were compared, histologic type, tumor size, lymph node metastasis, and LDH levels were found to be significant factors in making a decision on PMRT for patients with T1-2 tumors and 1-3 lymph node metastasis. But, OS and DFS were not different when the two groups were compared. This study results indicate that pretreatment blood parameters were not important in the prognosis. We attribute this to the fact that we worked with a relatively small and nonhomogeneous group of patients. While conducting breast cancer-related survival analysis, larger groups, new prognostic-predictive factors, new treatment regimens, and new risk stratifications are needed.

\section{Disclosure}

The authors report no conflicts of interest in this work.

\section{References}

1. Belkacémi Y, Fourquet A, Cutuli B, et al. French Expert Review Board of Nice/Saint-Paul de Vence. Radiotherapy for invasive breast cancer: guidelines for clinical practice from the French expert review board of Nice/ Saint-Paul de Vence. Crit Rev Oncol Hematol. 2011;79(2):91-102.

2. Truong PT, Olivotto IA, Kader HA, Panades M, Speers CH, Berthelet E. Selecting breast cancer patients with $\mathrm{T} 1-\mathrm{T} 2$ tumors and one to three positive axillary nodes at high postmastectomy locoregional recurrence risk for adjuvant radiotherapy. Int J Radiat Oncol Biol Phys. 2005; 61(5):1337-1347.

3. Geng W, Zhang B, Li D, Liang X, Cao X. The effects of ECE on the benefits of PMRT for breast cancer patients with positive axillary nodes. $J$ Radiat Res. 2013;54(4):712-718.

4. Yang PS, Chen CM, Liu MC, et al. Radiotherapy can decrease locoregional recurrence and increase survival in mastectomy patients with $\mathrm{T} 1$ to $\mathrm{T} 2$ breast cancer and one to three positive nodes with negative estrogen receptor and positive lymphovascular invasion status. Int J Radiat Oncol Biol Phys. 2010;77(2):516-522.

5. Abdulkarim BS, Cuartero J, Hanson J, Deschênes J, Lesniak D, Sabri S. Increased risk of locoregional recurrence for women with T1-2N0 triplenegative breast cancer treated with modified radical mastectomy without adjuvant radiation therapy compared with breast-conserving therapy. J Clin Oncol. 2011;29(21):2852-2858.

6. Moo TA, McMillan R, Lee M, et al. Selection criteria for postmastectomy radiotherapy in t1-t2 tumors with 1 to 3 positive lymph nodes. Ann Surg Oncol. 2013;20(10):3169-3174.

OncoTargets and Therapy

\section{Publish your work in this journal}

OncoTargets and Therapy is an international, peer-reviewed, open access journal focusing on the pathological basis of all cancers, potential targets for therapy and treatment protocols employed to improve the management of cancer patients. The journal also focuses on the impact of management programs and new therapeutic agents and protocols on
7. Huang CJ, Hou MF, Chuang HY, et al. Comparison of clinical outcome of breast cancer patients with T1-2 tumor and one to three positive nodes with or without postmastectomy radiation therapy. Jpn J Clin Oncol. 2012;42(8):711-720.

8. Nagao T, Kinoshita T, Tamura N, Hojo T, Morota M, Kagami Y. Locoregional recurrence risk factors in breast cancer patients with positive axillary lymph nodes and the impact of postmastectomy radiotherapy. Int J Clin Oncol. 2013;18(1):54-61.

9. Moo TA, McMillan R, Lee M, et al. Impact of molecular subtype on locoregional recurrence in mastectomy patients with $\mathrm{T} 1-\mathrm{T} 2$ breast cancer and 1-3 positive lymph nodes. Ann Surg Oncol. 2014;21(5): $1569-1574$

10. McBride A, Allen P, Woodward W, et al. Locoregional recurrence risk for patients with $\mathrm{T} 1,2$ breast cancer with 1-3 positive lymph nodes treated with mastectomy and systemic treatment. Int $J$ Radiat Oncol Biol Phys. 2014;89(2):392-398.

11. Edge SB, Byrd DR, Compton CC, Fritz AG, Greene FL, Trotti A, editors AJCC Cancer Staging Manual. 7th ed. New York: Springer; 2010.

12. Ragaz J, Jackson SM, Le N, et al. Adjuvant radiotherapy and chemotherapy in node-positive premenopausal women with breast cancer. N Engl J Med. 1997;337(14):956-962.

13. Gnant M, Harbeck N, Thomssen C. St. Gallen 2011: Summary of the Consensus Discussion. Breast Care (Basel). 2011;6(2):136-141.

14. Koscielny S, Tubiana M, Le M, et al. Breast cancer: relationship between the size of the primary tumor and the probability of metastatic dissemination. Br J Cancer. 1984;49(6):709-715.

15. Gebauer G, Fehm T, Lang N, Jäger W. Tumor size, axillary lymph node status and steroid receptor expression in breast cancer: prognostic relevance 5 years after surgery. Breast Cancer Res Treat. 2002;75(2):167-173.

16. Carter C, Allen C, Henson D. Relation of tumor size, lymph node status and survival in 24,740 breast cancer cases. Cancer. 1989;63(1):181-187.

17. Shen H, Yuan J, Yang Y, et al. Prognostic analysis in a Chinese population with T1-2N1 breast cancer: did patients with 1 or 2, and 3 positive axillary lymph nodes have similar survival outcomes? J Surg Oncol. 2015;112(6):569-574.

18. Recht A, Gray R, Davidson NE, et al. Locoregional failure 10 years after mastectomy and adjuvant chemotherapy with or without tamoxifen without irradiation: experience of the Eastern Cooperative Oncology Group. J Clin Oncol. 1999;17(6):1689-1700.

19. Wallgren A, Bonetti M, Gelber RD, et al. Risk factors for locoregional recurrence among breast cancer patients: results from International Breast Cancer Study Group trials I through VII. J Clin Oncol. 2003; 21(7):1205-1213.

20. Kunkler IH, Canney P, van TG. Elucidating the role of chest wall irradiation in intermediate risk breast cancer: the MRC/EORTC SUPREMO trial. Clin Oncol (R Coll Radiol). 2008;20(1):31-34.

21. Overgaard M, Hensen PS, Overgaard J, et al. Post operative radiotherapy in high-risk premenopausal women with breast cancer who receive adjuvant chemotherapy: Danish Breast Cancer Cooperative Group 82b Trial. N Engl J Med. 1997;337(14):949-955.

22. Cihan YB, Arslan A, Cetindag MF, Mutlu H. Lack of prognostic value of blood parameters in patients receiving adjuvant radiotherapy for breast cancer. Asian Pac J Cancer Prev. 2014;15(10):4225-4231.

23. Petekkaya I, Aksoy S, Roach EC, et al. Impact of inflammatory markers on the prognosis of patients with operable breast cancer. J BUON. 2014; 19(3):673-680. patient perspectives such as quality of life, adherence and satisfaction. The manuscript management system is completely online and includes a very quick and fair peer-review system, which is all easy to use. Visit http://www.dovepress.com/testimonials.php to read real quotes from published authors. 